\title{
Actividad física y calidad de vida por parte de los pacientes de la atención primaria de la ciudad de Terrassa \\ Physical activity and quality of life by patients of primare care of the city of Terrassa
}

\author{
M.Paola Mastrantonio, Oscar Coduras \\ EUNCET Business School (España), Centro adscrito a la Universitat Politécnica de Catalunya (España)
}

\begin{abstract}
Resumen. En el artículo se describen y analizan los resultados de un estudio realizado para investigar la relación entre la práctica físico-deportiva y la calidad de vida percibida (CVP). La muestra del estudio estuvo integrada por un colectivo de ciudadanos (968), pacientes de Atención Primaria, entrevistados mientras se encontraban en las salas de espera, de cuatro Centros de Atención Primaria (CAP) de la ciudad de Terrassa. Los objetivos del estudio fueron: 1) Evaluar la relación entre la percepción de calidad de vida y la actividad físico-deportiva; 2) Verificar si, en tal percepción, hay diferencias significativas entre hombres y mujeres. Las conclusiones fueron las siguientes: Existe una percepción mejor de la calidad de vida entre los usuarios que realizan actividad físico-deportiva que entre los pacientes sedentarios, sea la actividad moderada o intensa. Las mujeres que realizan actividad físico-deportiva mejoran su percepción de Calidad de Vida en mayor grado que los hombres. Las mujeres mejoran su CVP en el ámbito social y los hombres en el ámbito físico, por lo que la práctica deportiva es una fuente de la socialización de las mujeres.
\end{abstract}

Palabras clave: Actividad físico-deportiva, Calidad de vida, Pacientes, sexo.

\begin{abstract}
The article describes and analyzes the results of a study carried out to investigate the relationship between physicalsports practice and perceived quality of life (QOL). The study sample was made up of a group of citizens (968), Primary Care patients, interviewed while they were in the waiting rooms of four Primary Care Centers (CAP) in the city of Terrassa. The objectives of the study were: 1) To evaluate the relationship between the perception of quality of life and physical-sports activity; 2) Verify if, in such perception, there are significant differences between men and women. The conclusions were as follows: There is a better perception of the quality of life among users who perform physical-sports activity than among sedentary patients, be it moderate or intense activity. Women who perform physical-sports activity improve their perception of Quality of Life to a greater degree than men.Women improve their CVP in the social sphere and men in the physical sphere, so sports practice is a source of women's socialization.
\end{abstract}

Palabras clave en inglés: Physical Activity, Quality of Life, patients, gender.

\section{Introducción}

En los últimos años, la práctica regular de la actividad físico-deportiva se ha incrementado de manera exponencial. El principal motivo de este aumento ha sido la importancia creciente que la población le da a la salud y, adicionalmente, a la estética, y su consiguiente necesidad y deseo de realizar actividad física o deportiva en cualquiera de sus acepciones. Según la encuesta de hábitos deportivos de España, elaborada por la División de Estadísticas y Estudios del Ministerio de Cultura y Deporte (2015), el 53,5\% de la población española realizaba ejercicio físico de manera regular.

Consecuentemente, el número de artículos referidos al tema ha aumentado considerablemente, con ob-

Fecha recepción: 21-07-21. Fecha de aceptación: 10-12-21

María Paola Mastrantonio Ramos

mpmastrantonio@euncet.es jetivos bastante diversos. Por un lado, están los estudios que pretenden caracterizar a los usuarios practicantes de la actividad físico-deportiva y poder, con ello, adecuar los centros a la diversidad de intereses que tienen estos usuarios o clientes (Nuviala, Teva-Villén, PérezOrdás, Grao-Cruces, Tamayo \& Nuviala 2014), (Águila, Sicilia, Mayor \& Orta, 2009). Otros estudios miden la satisfacción de los usuarios en referencia a los servicios que ofrecen los centros (Elasri-Ejjaberi,Triadó-Ivern \& Aparicio-Chueca, 2016), (Aparicio-Chueca, ElasriEjjaberi \&Triadó-Ivern, 2018) en términos de calidad de los servicios prestados.

Dado que el número de practicantes ha aumentado, también existen diversos estudios de segmentación de clientes para identificar sus características, adoptar estrategias de fidelización y mejorar la gestión de los centros deportivos. Por último y sin abarcar todos los objetivos posibles, otros artículos (Nuviala, 2014), (Leytón, García, Fuente \& Jiménez, 2018) analizan aspectos 
motivacionales de la práctica deportiva. Entre otros están (Sicilia, Águila, Mayor, Orta \& Moreno, 2009), (Leytón, García, Fuentes \& Jiménez, 2018).

Sin embargo, el estudio de la práctica regular de actividad físico-deportiva y sus efectos en la percepción de la calidad de vida es más infrecuente, aunque todos ellos confirman la relación directa entre ambos conceptos, especialmente en la población de mayor edad (Quesada \& Gómez-López, 2017), (García-Pascual, Silla-Merchán, Mundina \& Escamilla, 2016), (Silla, Calabuig \& Añó, 2014), y (Villarreal, Moncada, Ochoa \& Hall, 2021). Todos concluyen que, si las personas hacen actividad físico-deportiva de manera regular, perciben una mejora en su calidad de vida. Estos artículos procuran descubrir los aspectos deficitarios de la vida de los adultos, como el sedentarismo y los problemas de salud que conlleva la falta de ejercicio físico. (Salinas, Cocca, Mohamed \& Viciana, 2010), (García y Froment, 2018).

Al mismo tiempo, el concepto de calidad de vida se ha estudiado asaz en muchos ámbitos y, en particular, en el ámbito de la salud, poniendo de manifiesto la amplia gama de acepciones del término (Schwartzmann, 2003), (Ardila, 2003), (Urzúa \& Caqueo-Urízar, 2012). La larga lista de definiciones de calidad de vida se refleja en el artículo de Velarde-Jurado \& Ávila-Figueroa (2002), en el cual se realiza una revisión pormenorizada de la evaluación del concepto de calidad de vida. La dificultad de buscar una definición sólida y única de calidad de vida radica en que son variadas las conceptualizaciones que utilizan el concepto como, por ejemplo, en el ambiente laboral (Reimel, 2015), (Fernández, Cuairan \& Curbelo, 2016), en la adolescencia (Higuita-Gutiérrez \& Cardona-Arias, 2015), (Martínez, Vergel \& Zafra, 2015), en el adulto mayor (Froment \& García-González, 2018) y, con mayor frecuencia, en personas con alguna patología, cuestión que se aborda en este trabajo. La calidad de vida es un concepto multidimensional y eminentemente cualitativo (Velarde, 2002).

Según Motilla, Sánchez-Oro, Curiel, Rodríguez, López \& López de Castro (2009), el 69,7\% de las personas que se visitan con su médico de familia lo hacen por tener alguna enfermedad crónica, por lo que la población de este estudio refleja esta realidad.

El principal objetivo del estudio que ha dado lugar al presente artículo ha sido el de corroborar la existencia de una relación indisoluble entre la valoración de la calidad de vida percibida (CVP) y la práctica de actividades físico-deportivas, por parte de los ciudadanos que se encontraban en algún Centro de Atención Primaria
(CAP) de la ciudad de Terrassa, provincia de Barcelona. El equipo investigador decidió utilizar el concepto de calidad de vida percibida de la OMS, definida como la percepción del individuo sobre su posición en la vida, en el contexto de la cultura, el sistema de valores en que vive, en relación con sus objetivos, expectativas, estándares y preocupaciones (Carrasco, 1998, 2012), (Espinoza, Osorio, Torrejón, Carrasco \& Bunout, 2011). Es un concepto integrador y multidimensional, ya que divide la calidad de vida en cuatro dimensiones, dominios o áreas: Físico, Psicológico, Social y Ambiental.

La OMS (2010) elaboró un instrumento que mide dichas dimensiones llamado Wordl Health Organization Quality of Life Questionnaire. El Cuestionario WHOQOL-BREF no sólo determina la calidad de vida de los encuestados sino, también, todas sus dimensiones (física, psicológica, social y ambiental), así como la percepción que tiene el encuestado de su propia calidad de vida.

Para el estudio, se plantearon las siguientes hipótesis:

Hipótesis 1: las personas de la muestra que realizan actividad físico-deportiva perciben significativamente mejor su calidad de vida que las personas que no la practican.

Hipótesis 2: las mujeres y hombres que realizan actividad física aumentan su CVP cuando la cantidad de ejercicio a la semana es mayor a 150 minutos (lo sugerido por la OMS).

Hipótesis 3: Si se analizan los motivos por los cuales hombres y mujeres realizan actividad física, no hay diferencias significativas entre ambos sexos.

Hipótesis 4: Las mujeres que no hacen actividad física y que son mayores de 55 años son las que peor perciben su calidad de vida, en relación con el resto de la muestra.

\section{Material y método}

\section{Diseño}

A través del Instituto de Estadística de Cataluña (IDESCAT), se estableció el número de habitantes de Terrassa en 2019, que era de 223.627 personas. Se eliminaron los menores de 18 años y se realizó un muestreo por conveniencia, seleccionándose previamente los CAP de mayor afluencia, determinando, inicialmente, un + / - 5\% de margen de error y un nivel de confianza del 95\%, es decir, una muestra de 400 individuos. Dado el buen trabajo realizado por los encuestadores con sus IPAD preparadas para el trabajo, se consiguió un núme- 
ro de encuestas muy superior (968) al mínimo necesario para el alcance de la fiabilidad pretendida (400), de acuerdo con la siguiente fórmula de cálculo de la muestra necesaria para poblaciones no finitas:

$$
\mathrm{n}=\frac{4 \mathrm{p}(1-\mathrm{p})}{\mathrm{K}^{2}}
$$

donde $\mathrm{p}=0,5$ (dispersión máxima) y $\mathrm{K}=0,05$ (margen de error). Dada la muestra alcanzada (968 individuos), el margen de error disminuyó hasta el +/ - 3,2\%.

\section{Participantes}

Así pues, finalmente, la muestra del estudio estuvo compuesta por 968 sujetos (573 mujeres y 395 hombres), de edades comprendidas entre los 18 y los 84 años que, al ser entrevistados, esperaban a ser atendidos por su médico de familia en 4 Centros de Atención Primaria (CAP) de la ciudad de Terrassa.

Se consideraron estos CAPs por estar ubicados en 4 distritos distintos de la ciudad de Terrassa (centro de la ciudad, norte, sur y oeste). Adicionalmente, se siguieron las sugerencias de los profesionales del Hospital Mutua de Terrassa, quienes indicaron que estos centros eran los más concurridos de la ciudad. Dichos CAPs, en el momento de la realización del trabajo de campo, estaban abiertos todo el día y los encuestadores ( 2 en cada centro periférico y 4 en el central) estuvieron realizando encuestas durante 8 horas (4 en las mañanas y 4 en las tardes), durante 4 semanas.

\section{Instrumentos}

El cuestionario final fue configurado utilizando las siguientes escalas:

El Cuestionario WHOQOL-BREF (Wordl Health Organization Quality of Life Questionnaire), que se centra en la CVP y que está compuesto por 26 preguntas, una pregunta por cada una de las facetas contenidas en el WHOQOL-100.

La escala WHOQOL-BREF, que genera un perfil de CVP de cuatro áreas o ámbitos: física (salud), psicológica, relaciones sociales y ambiente (Skevington, Lotfy \& O’Connell KA, 2004), (Cardona \& Higuita, 2014). Sobre la validación de este instrumento en el idioma castellano, puede afirmarse que fue reportada en España (Lucas-Carrasco, 2012).

La escala de la Encuesta de Hábitos Deportivos del Ministerio de Educación, Cultura y Deporte (2020), que se realiza a nivel del conjunto del Estado cada quinquenio. Se consideraron 15 preguntas sobre actividad físico-deportivas, relacionadas con el tiempo dedicado, los días de actividad física, la clasificación de actividad física que realizan, el grado de intensidad (moderada o intensa), días preferidos de entrenamiento, etc.

La escala de motivos por los que se hace actividad física, Motives for Physical Activity Measure-Revised (MPMR-R) de Ryan, Frederick, Lepes Rubio, y Sheldon, (1997), traducida y adaptada al español por Moreno, Cervelló y Martínez (2007).

El instrumento completo ha mostrado una consistencia alta, registrando un coeficiente Alfa de Cronbach de á $=0,839$.

\section{Procedimiento}

Atendiendo a los objetivos e hipótesis de la investigación y los recursos disponibles, el trabajo de campo fue realizado durante 4 semanas del mes de noviembre de 2019, distribuyéndose los encuestadores en 2 turnos: mañana y tarde. Para garantizar la homogeneidad de las condiciones en el procedimiento de recogida de datos, todos los encuestadores recibieron una formación previa de dos horas en técnicas de encuesta y observación, así como sobre la aplicación del formato de encuesta, estructurada mediante dispositivos electrónicos (IPADs). Los encuestadores fueron entrenados mediante un modelo instruccional, basado en simulaciones de situaciones breves.

En general, las personas encuestadas necesitaron entre 15 y 20 minutos para responder a todas las preguntas del cuestionario. Todas las personas encuestadas aceptaron participar voluntariamente y fueron informadas de la confidencialidad de los datos facilitados. La única dificultad que hubo fue que, pacientes que estaban siendo entrevistados, debían pasar a la consulta, por lo que quedaron a medias los cuestionarios y hubo que eliminarlos (97 casos).

\section{Resultados}

\section{Resultados generales}

Del total de los sujetos encuestados, el 34,4 \% (334 personas) manifestaron realizar actividad físico-deportiva, siendo un 65,5\%, el porcentaje de quienes manifestaron no realizar tal práctica (635 personas).

Este resultado obtenido sobre el total de sujetos que no practican actividad físico-deportiva es notablemente elevado comparado con que el registrado de los datos nacionales de la encuesta de hábitos deportivos de España, según la cual el 53,5\% de la población española realiza ejercicio físico de manera regular (2015), siendo un $46,5 \%$ el porcentaje de quienes no lo realizan. Sin em- 
bargo, esta diferencia es sostenible con estudios relacionados con pacientes que asisten a consultas con el médico de familia, como el de Campo, Fernández, Azorín, Ossa, Auñón \& Párraga (2019).

La tabla 1 muestra la comparación de medias de las valoraciones de Calidad de Vida (OMS) y de cada uno de sus componentes (dominios) de las personas que realizan actividad físico-deportiva, en comparación con las que no la realizan. Como puede observarse, las diferencias entre quienes realizan y no realizan actividad físico-deportiva son notablemente significativas, con un $\mathrm{p}$ valor $<.005$. Ello se puede resumir diciendo que los pacientes que practican actividad física perciben mejor su CV que los que no la practican. Con ello, se comprueba la hipótesis 1 .

Tabla 1.

Número total de personas de la muestra que hacen o no actividad fisica y calidad de vida ( $\mathrm{N}=969)$

\begin{tabular}{lccccc}
\hline & ¿Practica deporte? & $\mathrm{N}$ & Media & Desviación típica & Sig. Bilateral \\
\hline Dominio1 Físico & $\mathrm{si}$ & 380 & 14,82 & 2,003 & 0,00 \\
& $\mathrm{no}$ & 588 & 13,79 & 2,068 & 0,00 \\
Dominio2 Psicológico & $\mathrm{si}$ & 380 & 15,12 & 1,649 & 0,00 \\
& $\mathrm{no}$ & 588 & 14,22 & 1,814 & 0,00 \\
Dominio3 Social & $\mathrm{si}$ & 380 & 15,92 & 3,024 & 0,00 \\
& $\mathrm{no}$ & 588 & 14,42 & 3,151 & 0,00 \\
Dominio4 ambiental & $\mathrm{si}$ & 380 & 15,16 & 2,018 & 0,00 \\
& $\mathrm{no}$ & 588 & 14,25 & 2,142 & 0,00 \\
CVTOTAL & $\mathrm{si}$ & 380 & 15,25 & 1,794 & 0,00 \\
& $\mathrm{no}$ & 588 & 14,17 & 1,854 & 0,00 \\
\hline
\end{tabular}

\section{Diferencias por sexo}

Si consideramos a los practicantes deportivos, las mujeres practican menos tiempo que los hombres, en todos los grupos de edad, lo que se muestra en el gráfico 1. Es más, si nos decidimos a tomar la sugerencia de la OMS de practicar cuanto menos 150 minutos a la semana para tener una vida saludable, el único grupo de edad de mujeres que cumple con esta recomendación es el de mujeres menores de 34 años; en cambio, en el caso de los hombres, lo cumplen todos los grupos a excepción del grupo de mayores de 64 años.

Sin embargo, si medimos calidad de vida de hombres y mujeres que practican más o menos 150 minutos, no existen diferencias significativas como se expre-

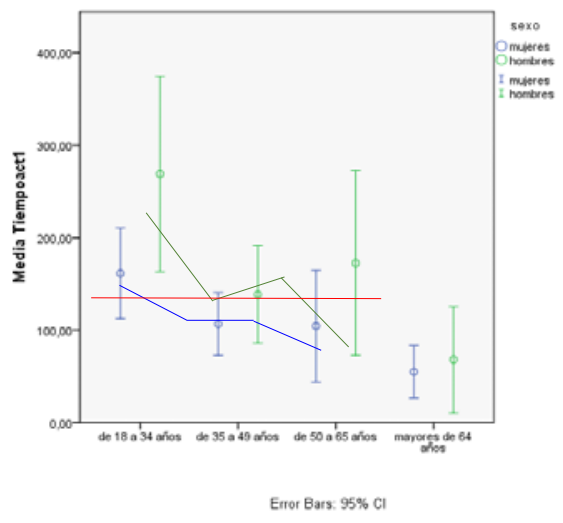

Gráfico1. Comparación de tiempo de actividad fisica entre hombres y mujeres por grupo etario en minutos sa en la tabla 2. Se realizó la prueba de Levene para muestras independientes, donde la significancia bilateral es mayor que 0,05. No se cumple la hipótesis 2 .

También ocurre que el tipo de actividad ya sea esta intensiva o moderada, no afecta la calidad de vida de las personas. Al parecer, solamente el hecho de practicar alguna actividad físico-deportiva cambia su percepción de calidad de vida, independientemente de la duración y la intensidad de ésta.

Tabla 2

\begin{tabular}{|c|c|c|c|c|c|c|c|}
\hline & tope 150 & \multirow{2}{*}{$\mathrm{N}$} & Media & \multicolumn{2}{|r|}{ Desviación típ. } & \multicolumn{2}{|c|}{ Error típ. de la media } \\
\hline \multirow[t]{4}{*}{$\overline{\text { CVTOTAL }}$} & $<150$ minutos & & 15,0917 & \multicolumn{2}{|r|}{1,72260} & \multicolumn{2}{|c|}{, 15725} \\
\hline & $>=150$ minutos & 258 & 15,3440 & \multicolumn{2}{|r|}{1,82760} & &, 11378 \\
\hline & & & & \multicolumn{4}{|c|}{$\begin{array}{c}\text { Prueba de Levene para la igualdad de } \\
\text { varianzas }\end{array}$} \\
\hline & & & & F & Sig. & $\mathrm{gl}$ & Sig. (bilateral) \\
\hline \multirow[t]{2}{*}{ CVTOTAL } & Se han asumid & variar & iguales & 0,554 & $40,457-1,311$ & 332 & 0,191 \\
\hline & No se han asur & hido va & zzas iguales & & $-1,309$ & 144,916 & 0,193 \\
\hline
\end{tabular}

Respecto de los motivos por los cuales las personas realizan actividad física, no hay diferencias significativas entre hombres y mujeres, a excepción del motivo social. Las mujeres dan mayor importancia que los hombres a realizar actividad física por motivos sociales. Ello, en este motivo específico, contradice la hipótesis 3.

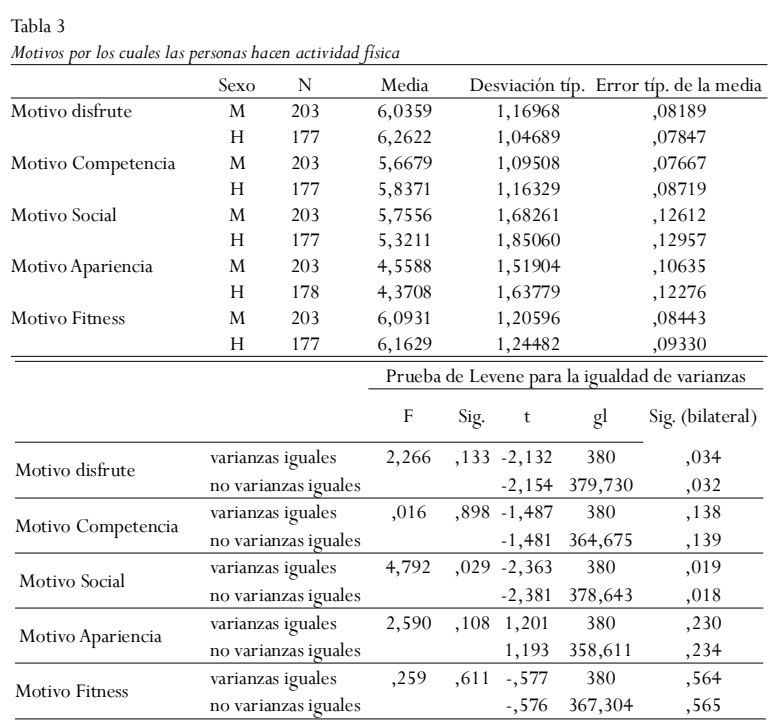

En las tablas 4.1 y 4.2 se ha agregado una última columna que resume las diferencias, en puntos, de la valoración de calidad de vida entre los que practican o no actividad físico-deportiva. Se puede concluir que las mujeres presentan diferenciales mayores que los hombres, tanto con respecto a cada uno de los dominios de CVP, como con respecto a la calidad de vida total (mujeres $=4,75$ puntos y hombre $=3,65$ puntos).

Por consiguiente, la falta de ejercicio afecta más negativamente en su CVP a las mujeres que a los hombres. Cualquier intervención para mejorar la CVP de los pacientes debe ir orientada preferentemente a mujeres. 


\begin{tabular}{|c|c|c|c|c|c|}
\hline & ¿Practica AF o deporte? & $\mathrm{N}$ & Media & Desviación típ. & Dif. Medias \\
\hline \multirow[t]{2}{*}{ Dominio Físico } & si & 203 & 15,01 & 1,984 & 1,1 \\
\hline & no & 370 & 13,91 & 2,009 & \\
\hline \multirow[t]{2}{*}{ Dominio Psicológico } & si & 203 & 15,19 & 1,605 & 0,98 \\
\hline & no & 371 & 14,21 & 1,809 & \\
\hline \multirow[t]{2}{*}{ Dominio Social } & si & 203 & 15,97 & 2,985 & 1,76 \\
\hline & no & 370 & 14,30 & 3,269 & \\
\hline \multirow[t]{3}{*}{ Dominio ambiental } & si & 203 & 15,27 & 2,006 & 0,91 \\
\hline & no & 371 & 14,36 & 2,141 & \\
\hline & & & & total & 4,75 \\
\hline \multicolumn{6}{|l|}{ Tabla 4.2} \\
\hline \multicolumn{6}{|c|}{ Calidad deVida, actividad fisica y hombres } \\
\hline & ¿Practica AF o deporte? & $\mathrm{N}$ & Media & Desviación típ. & Dif. medias \\
\hline \multirow[t]{2}{*}{ Dominio Físico } & si & 177 & 14,66 & 2,010 & 0,94 \\
\hline & no & 218 & 13,72 & 2,101 & \\
\hline \multirow[t]{2}{*}{ Dominio Psicológico } & si & 177 & 15,05 & 1,688 & 0,82 \\
\hline & no & 218 & 14,23 & 1,820 & \\
\hline \multirow[t]{2}{*}{ Dominio Social } & si & 177 & 15,89 & 3,066 & 1,0 \\
\hline & no & 218 & 14,89 & 3,081 & \\
\hline \multirow[t]{3}{*}{ Dominio ambiental } & si & 177 & 15,07 & 2,028 & 0,89 \\
\hline & no & 218 & 14,18 & 2,143 & \\
\hline & & & & total & 3,65 \\
\hline
\end{tabular}

A partir de estos hallazgos y relacionándolo con otros ya mencionados (Mastrantonio, Coduras, 2020), se segmentó la muestra para hallar a aquellas mujeres que presentaron valoraciones más bajas de su calidad de vida. Se segmentó la muestra total de mujeres mayores de 55 años, seleccionando mujeres que no hacen actividad físico-deportiva y que, efectivamente, perciben comparativamente peor su calidad de vida. Sin embargo, el grupo total de 55 años es muy pequeño con un total de 166 mujeres mayores de 55 años, de las cuales sólo el $22,9 \%$ realiza actividad física de manera habitual. Se cumple la hipótesis 4.

\begin{tabular}{|c|c|c|c|c|c|}
\hline & ¿Practica AF o deporte? & $\mathrm{N}$ & Media & Desviación típ. & Dif. Medias \\
\hline \multirow[t]{2}{*}{ Dominio Físico } & si & 38 & 14,28 & 1,814 & 1,27 \\
\hline & no & 128 & 13,01 & 1,956 & \\
\hline \multirow[t]{2}{*}{ Dominio Psicológico } & $\mathrm{si}$ & 38 & 15,00 & 1,692 & 1,13 \\
\hline & no & 128 & 13,87 & 1,607 & \\
\hline \multirow[t]{2}{*}{ Dominio Social } & si & 38 & 15,31 & 3,111 & 1,44 \\
\hline & no & 128 & 13,76 & 2,689 & \\
\hline \multirow[t]{3}{*}{ Dominio ambiental } & si & 38 & 15,23 & 1,880 & 1,47 \\
\hline & no & 128 & 13,82 & 1,789 & \\
\hline & & & & total & 5,31 \\
\hline
\end{tabular}

\section{Discusión}

La relación entre calidad de vida y actividad física es innegable puesto que, según los resultados obtenidos, las personas que realizan actividad física de manera habitual perciben mejor su calidad de vida que las que refieren no hacer ninguna actividad físico-deportiva, lo que se ha puesto claramente de manifiesto a pesar de que la muestra del estudio haya estado integrada por usuarios en espera de ser atendidos por su médico de familia, un colectivo especial por ser pacientes de atención primaria. Tal como dice Molina (2018), la actividad física y los deportes son un vehículo inestimable para la calidad de vida de cualquier colectivo.

Todas las personas que realizan actividad física mues- tran satisfacción con su calidad de vida. Además, la cantidad de ejercicio (en minutos) que hagan y la intensidad de éste (moderado o intenso), no afecta a su percepción de calidad de vida. Las diferencias son significativas sólo cuando se comparan las personas que practican con las que no practican, lo que indica que el sólo hecho de practicar alguna actividad física, aunque sea moderada o pocos minutos a la semana, genera en las personas un sentimiento positivo respecto de su calidad de vida.

Los datos conducen a validar la hipótesis 1 referida a que aquellas personas que realizan actividad físico-deportiva muestran mayor satisfacción con su calidad de vida que aquellas personas que no realizan actividad físico-deportiva, ya sean hombres o mujeres. Esta relación ha sido comprobada muchas veces por otros autores para distintos colectivos como Alarcón \& Abensur (2020), Nieto-López, García-Canto \& Rosa-Guillamón, (2020), García-Tascón, Mendaña-Cuervo, Sahelices-Pinto \& Margaz-González (2021), entre otros.

Continuando con el análisis, las mujeres tienden a percibir peor su calidad de vida que los hombres, es decir, si comparamos las mujeres que realizan práctica deportiva con las que no la realizan, el diferencial, en puntos, de CVP es mayor que si hacemos lo mismo en hombres. Por consiguiente, si se quiere mejorar la calidad de vida de los habitantes de Terrassa, en general, tendría que aplicarse una política al respecto dirigida a las mujeres, ya que el efecto sería mayor que entre los hombres, compensando el desequilibrio actual entre géneros.

Además de lo ya expuesto, otra de las cuestiones que pusieron de manifiesto los resultados del estudio, fue la demostración de que el diferencial mayor en los dominios (entre quienes hacen y quienes no hacen actividad física) se encuentra, sólo para las mujeres, en el ámbito social, es decir, que el ejercicio físico refuerza de manera significativa el área social de las mujeres, inclusive por encima del aspecto físico, tal como lo explican González-Hernández \& Martínez-Martínez (2020), Cancela, Vila, Sánchez-Lastra \& Varela (2021), habiéndose observado que las mujeres tienen una valoración media significativamente mayor que los hombres acerca de este aspecto.

En un artículo de Mastrantonio y Coduras, (2019) referido a ciudadanos deTerrassa en relación con la práctica deportiva, se concluyó que uno de los grupos de personas que debía estudiarse específicamente era el de las mujeres mayores de 55 años, ya que eran muy infrecuentes en los diversos grupos de personas que rea- 
lizan actividad física en los centros deportivos públicos de Terrassa.

Por ello, se analizó el caso particular de las mujeres mayores de 55 años. Se compararon las frecuencias entre practicantes y no practicantes de ejercicio físico, y se concluyó que, de manera significativa, las mujeres mayores de 55 años son las que peor perciben su calidad de vida, tal como se aprecia en el cuadro 5. En este punto, es interesante comparar estos resultados con los obtenidos por Elasri-Ejjaberi,Triadó-Ivern y AparicioChueca (2016) que realizaron un trabajo similar en Barcelona. Al segmentar por género, ellos encuentran un cluster de mujeres mayores de 60 años que asisten a los centros deportivos públicos de Barcelona. Según López, Irisarri y Fernández (2017), en las mujeres mayores de 50 años, el apoyo social es imprescindible para que ellas puedan realizar actividad física.

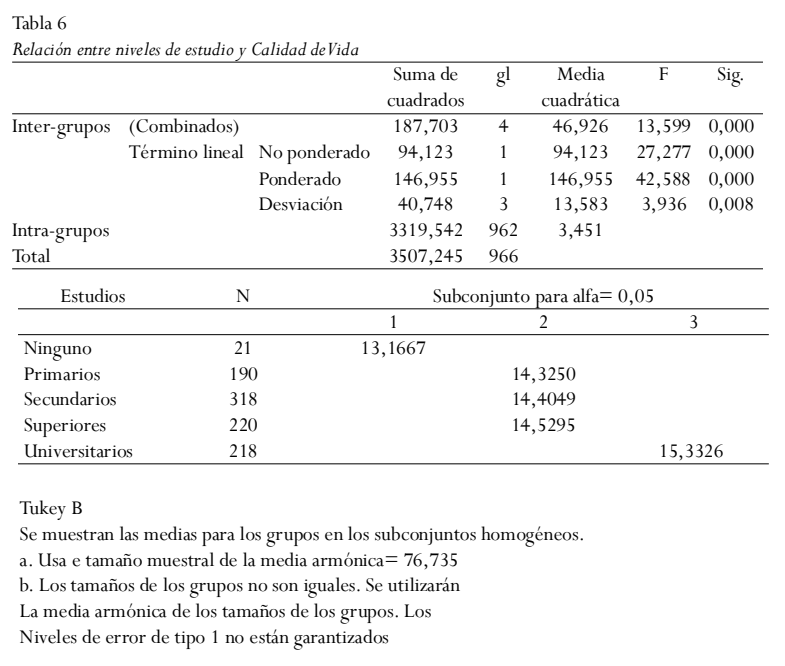

Otra de las cuestiones adicionales a las hasta aquí mencionadas y extraída de los resultados, fue identificar que los años de educación de los individuos también es una variable relacionada con la propensión a la práctica deportiva y, por consiguiente, con la CVP, ya que las personas que tienen menores niveles de estudios realizan menos actividad físico-deportiva que quienes tienen un recorrido educativo más largo, por lo que puede afirmarse que la prolongación de la educación contribuye a crear una conducta de adhesión a la práctica deportiva.

\section{Conclusiones}

Los resultados encontrados sobre la relación entre Calidad de Vida y actividad físico-deportiva confirman la relación estrecha que hay entre estas dos variables. Cuando las personas hacen ejercicio físico, mejoran la percepción de su calidad de vida. En el caso de las mujeres es el ámbito social el que mejora más, de manera significativa, por encima del dominio físico (asociado a la salud) que podría ser lo esperable. Ello ocurre porque las mujeres ven en el deporte una actividad que les permite socializar: prefieren actividad física en grupos y al aire libre. En el caso de los hombres, el dominio o ámbito físico aumenta de manera significativa por encima del dominio o ámbito social. La intensidad del ejercicio y la duración de éste, no son significativos para considerar la CVP.

Finalmente, cabe añadir una última cuestión entre las que se han puesto de manifiesto en el tratamiento de esta muestra, y es el hecho de que los hombres practican actividad físico-deportiva mucho más tiempo que las mujeres, como se puede apreciar en el gráfico 1. Esto se podría explicar porque las mujeres en edad reproductiva tienden a priorizar la familia y limitan su adhesión a la actividad físico-deportiva, mientras que los hombres, en las mismas edades, mantienen la práctica deportiva por encima de los 150 minutos semanales. En definitiva, es un desequilibrio más en detrimento de la práctica deportiva de las mujeres, de su nivel de sanidad vinculada a ésta y de su percepción de CVP.

Como posibles líneas de investigación posteriores para profundizar, se han descubierto como mínimo dos cuestiones interesantes:

Una se refiere a las características de la muestra: las personas que se encuentran en una sala de espera de un centro de atención primaria esperando la consulta con el médico de familia asisten para resolver distintas necesidades (diagnóstico, prescripción de medicamentos, solicitud de especialista, seguimiento, etc.). Según Motilla, et. al, (2009) el 55,7\% son mujeres y el 69,7\% poseen una enfermedad crónica por lo que sería interesante identificar y, en su caso, diseñar alternativas de programas de actividad física especialmente concebidos para estos colectivos, que fueran compatibles con sus problemas de salud, con los que mejorar su calidad de vida, ya que la actividad física es un elemento fundamental para mantener un estado óptimo de salud de las personas mayores (Salinas, Cocca, Mohamed, Viciana, 2010). Diseñar programas especiales sugeridos por los médicos de familia ayudarían a los pacientes a mejorar su percepción de CV.

Asimismo, con base en la importancia manifiesta del motivo o ámbito social para el colectivo de mujeres, podría ser conveniente explorar el diseño de iniciativas de incentivación de la práctica de las actividades físico-deportivas poniendo énfasis en el aspecto relacional 
de tales actividades, es decir, en el ámbito social, en especial para el colectivo de mujeres mayores de 55 años que no realizan actividad física: actividades al aire libre y en grupos que les permitan socializar a la vez que habituarse paulatinamente a la actividad físico-deportiva.

\section{Referencias}

Alarcón, R. \& Abensur, C. (2020). Una estrategia para disminuir el deterioro del sistema muscular y mejorar la calidad de vida en personas de la tercera edad, en los distritos de Pachacamac. Ciencia y Desarrollo, vol.23 (2), 43-50

Águila, C., Sicilia, A., Mayor, J. \& Orta, A. (2009). Cultura posmoderna y perfiles de práctica en los centros deportivos municipales. Revista Internacional de Medicina y Ciencias de la Actividad Física y el Deporte, vol. 9 (33), 81-95.

Aparicio-Chueca, P., Elasri-Ejjaberi, A. \& Triadó-Ivern X. M. (2018). La satisfacción de los usuarios de actividades dirigidas de los centros deportivos municipales de Barcelona. Sport TK. Revista Euroamericana de Ciencias del Deporte, 7 (2), 27-33. Recuperado de https://doi.org/ $10.6018 /$ sportk.343071

Ardila, R. (2003). Calidad de vida: una definición integradora. Revista Latinoamericana de psicología, 35(2), 161-164.

Campo Giménez, M., Fernández Bosch, A., Azorín Ras, M., Ossa Moreno, M., Auñón Valero, B., \& Párraga Martínez, I. (2019). Frecuentación y calidad de vida de usuarios de consultas de Medicina de Familia. Revista Clínica de Medicina de Familia, 12(2), 50-60.

Cancela JM., Vila MH., Sánchez-Lastra M. \& Varela S. (2021). La socialización mejora la adherencia, pero no la fuerza, en programas de ejercicio físico con componente de fuerza en personas mayores, Revista andaluza de medicina del deporte, vol14(2), 103-107

Cardona-Arias, J.A. \& Higuita-Gutiérrez, L. F. (2014).Aplicaciones de un instrumento diseñado por la OMS para la evaluación de la calidad de vida. Revista Cubana de Salud Pública, 40(2), 175-189.

Carrasco, R. L. (2012). The WHO quality of life (WHOQOL) questionnaire: Spanish development and validation studies, Quality of Life Research, Volume 21(1), 161-165.

Elasri-Ejjaberi A., Triado-Ivern X. \& Aparicio-Chueca, P. (2016). Los usuarios de los centros deportivos públicos: una aproximación sobre hábitos deportivos y satisfacción. Revista de Psicología del Deporte, 15-18.

Fernández Araque, A., Cuairan Sola, M. \& Curbelo
Rodríguez, R. (2016). Calidad de vida profesional de enfermería en urgencias de un hospital de referencia. Enfermería Global. 15(2), 376-385. Recuperadodehttps://doi.org/10.6018/eglobal.15.2.235781.

Froment, F. \& García-González, A. (2018). Beneficios de la actividad física sobre la autoestima y la calidad de vida de personas mayores, Retos. Nuevas tendencias en educación fisica, deporte y recreación, 33, 3-9.

García, M. \& LLopis, R. (2011). Ideal democrático y bienestar personal: Encuesta sobre los hábitos deportivos en España 2010. Madrid: Centro de Investigaciones Sociológicas y Consejo Superior de Deportes.

García-Pascual, F., Silla-Merchán,A, Mundina, J. \& Escamilla, P. (2016). El efecto de variables de gestión en el bienestar subjetivo de usuarios de centros deportivos. Journal of Sports Economics \& Managament, 6(2), 99-110.

García, A, Froment, F. (2018). Beneficios de la actividad física sobre la autoestima y la calidad de vida de personas mayores. Retos. Nuevas tendencias en actividad fisica, deporte y recreación, 3-9.

García-Tascón, M., Mendaña-Cuervo, C., Sahelices-Pinto, C. \& Magaz-González,A. (2021). Repercusión en la calidad de vida, salud y práctica de actividad física del confinamiento por Covid-19 en España. Retos. Nuevastendencias en actividad fisica, deporte y recreación, vol. 42(21), 684695.

González-Hernandez, J., Martínez-Martínez, F. (2020). Prosocialidad y dificultades de socialización en la adolescencia. Influencias según sexo y práctica deportiva. Revista de Psicología del Deporte, vol. 29 (2), 117-124.

Hiquita-Gutiérrez, 1. \& Cardona-Arias, J. (2015). Concepto de calidad de vida en la adolescencia: una revisión crítica de la literatura, Revista CES Psicología, 8 (1), 155168.

IDESCAT, Instituto de Estadísticas de Cataluña, recuperado de: https: / / www.idescat.cat/emex/ ?id $=082798 \&$ lang $=$ es.

Jürgens, I. (2006). Práctica deportiva y percepción de calidad de vida, Revista Internacional de Medicina y Ciencias de la Actividad Física y del Deporte / International, 6 (22), 62-74. Leytón Román, M., García Matador, J., Fuentes García, J. P., \& Jiménez Castuera, R. (2017). Análisis de variables motivacionales y de estilos de vida saludables en practicantes de ejercicio físico en centros deportivos en función del género. Retos. Nuevas tendencias en Actividad física, deporte y recreación, 34, 166-171.

López, F. L., Irisarri, M. A. G. \& Fernández, M. J. (2017), Determinantes de la actividad física entre las personas de 50-70 años. Retos. Nuevas tendencias en actividad fisica, deporte y recreación, 31 (1),181-187. 
Lucas-Carrasco R. (2012). The WHO quality of life (WHOQoL) questionnaire: Spanish development and validation studies. Quality Life Res, 21, 161-165.

Martínez, J., Vargel, M. \& Zafra, S. (2015). Validez de instrumento para medir la calidad de vida en la juventud, Revista Logos. Ciencia y tecnología. 7 (1), 17-26.

Mastrantonio, M. P.\& Coduras, O. (2019). Actividad física y calidad de vida percibida en usuarios de centros deportivos públicos de Terrassa, Retos. Nuevas tendencias en educación física, deporte y recreación, 37, 427-433.

Ministerio de Educación, Cultura y Deporte. (2020). Encuesta de Hábitos Deportivos de España. Recuperado de https://www.culturaydeporte.gob.es.

Molina, F. (2018). Educación Física, Calidad de Vida y la Nueva Sociología de la infancia, Retos. Nuevas tendencias en educación fisica, deporte y recreación, 33, 69-73.

Moreno, J. A., Cervelló E., Martínez A. (2007). Validación de la Escala de Medida de los Motivos para la Actividad Física-Revisada en españoles: Diferencias por motivos de participación, Anales de psicología, vol. 23(1), 167-176.

Motilla, M., Sánchez-Oro, R., Curiel O., Rodríguez, B., López, M.J. \& López de Castro, F. (2009). ¿Qué cualidades valoran más los pacientes de Médicos de Familia?, Revista Clínica de Medicina Familiar, 2(6), 263-268.

Nieto-López, L., García-Canto, Eliseo. \& Rosa-Guillamón, A. (2021). Relación entre el nivel de forma física y la calidad de vida percibida relacionada con la salud en adolescentes del sureste de España. Revista de la Facultad de Medicina. 2020, vol.68(2), pp.533-540. Publicación electrónica 16 de enero de 2021.ISSN 0120-0011. https: / /doi.org/10.15446/revfacmed.v68n4.78052 .

Nuviala, R., Teva-Villén M., Pérez-Ordás, R., Grao-Cruces, A., Tamayo, J. A. \& Nuviala, A. (2014). Segmentación de usuarios de servicios deportivos, Retos. Nuevas tendencias en educación fisica, deporte y recreación, 25, 90-95.

Organización Mundial de la Salud, https: / /apps.who.int, Recomendaciones mundiales de la actividad física para la salud, Ediciones de la OMS, 2010.

Quesada, D. \& Gómez-López, M. (2017). Perfiles motivacionales de los usuarios de un centro deportivo público, Journal of sport and health research, 9 (1), 85-96.

Reimel S., (2015) Calidad de vida percibida de profesores universitarios: Dos muestras venezolanas, Revista Interamericana de Psicología ocupacional, Vol.13, 7-19.

Román, L., Matador, G., García, F. \& Castuera, J. (2018). Análisis de variables motivacionales y de estilos de vida saludables en practicantes de ejercicio físico en centros deportivos en función del género. Retos. Nuevas tendencias en educación fisica, deporte y recreación, 34, 166-171.

Ryan, R., Frederick, C., Lepes, D., Rubio, N., \& Sheldon,
K. (1997). Intrinsic Motivation and Exercise Adherence. Sport Psychology, 28, 335-354.

Salinas, F., Cocca, A., Mohamed, K., \& Viciana, J. (2010). Actividad Física y sedentarismo: repercusiones sobre la salud y calidad de vida de las personas mayores, Retos. Nuevas tendencias en educación física, deporte y recreación, 17, 126-129.

Schwartzmann, L. (2003). Calidad de vida relacionada con la salud: aspectos conceptuales. Ciencia y enfermería, 9(2), 09-21. Recuperado de https://dx.doi.org/10.4067/ s0717-95532003000200002.

Sicilia Camacho, Álvaro, Águila Soto, C., Muyor Rodríguez, J. M., Orta Cantón, A., \& Moreno Murcia, J.A. (2009). Perfiles motivacionales de los usuarios en centros deportivosmunicipales. Anales de Psicología, 25(1), 160-168. Recuperado de https:/ / revistas.um.es/analesps/ article/view/71621.

Silla, A., Calabuig,T. \& Añó, V. (2014). Emociones, satisfacción e intenciones futuras de los usuarios de actividades dirigidas de un centro deportivo. Journal of Sports Economics \& Management, (4), 22-38.

Skevington, S. M., Lotfy, M. \& O’Connell, K. 2. (2004). The World Health Organization's WHOQOL-BREF quality of life assessment: psychometric properties and results of the international field trial. A report from the WHOQOL group. Quality of life Research, 13(2), 299310.

Urzúa M, Alfonso, \& Caqueo-Urízar, Alejandra. (2012). Calidad de vida: Una revisión teórica del concepto. Terapia psicológica, 30(1), 61-71. Recuperado de https://dx.doi.org/10.4067/S071848082012000100006.

Velarde-Jurado, Elizabeth, \& Ávila-Figueroa, Carlos. (2002). Evaluación de la calidad de vida. Salud Pública de México, 44(4), 349-361. Recuperado de http:// w w w. s c i e l o . o r g. $\mathrm{m}$ x / scielo.php?script $=$ sci_arttext\&pid $=$ S003636342002000400009\&lng= ${ }_{\text {es\&tlng }}={ }_{\mathrm{es}}$.

Villareal, Mario., Moncada, José., Ochoa, Paulina. \& Hall, Arturo. (2021). Percepción de la calidad de vida del adulto mayor en México, Retos. Nuevas tendencias en educación fisica, deporte y recreación, 41, 480-484. 